\title{
Cardiac 'resynchronization' by a left lateral accessory pathway
}

\author{
Antonio O. Luque Lezcano, Jose M. Porres Aracama, \\ Francisco Garcia Urra, Vicente Urbistondo Ayestaran
}

Intensive Care Medicine, Arrhythmia Unit, Hospital Donostia, Gipuzkoa (Basque Country), Spain

\begin{abstract}
We present the case of a 21 year-old woman with unremarkable medical history except for tachycardia episodes. The ECG showed a delta wave pre-excitation by a left lateral pathway. One radiofrequency pulse temporarily terminated the accessory pathway conduction, revealing a nodal conduction with complete left bundle branch block. (Cardiol J 2012; 19, 5: 536-538)
\end{abstract}

Key words: Wolf-Parkinson-White, radiofrequency ablation, resynchronization

\section{Introduction}

We report the case of a healthy young woman with crisis of tachycardia and pre-excitation in basal electrocardiogram (ECG).

\section{Case report}

A 21 year-old woman with history of tachycardia was admitted to our service after three crises of chest palpitation (not registered) and an ECG showing pre-excitation by a left lateral pathway (Fig. 1). An electrophysiological study was performed.

After right femoral vein puncture, a $6 \mathrm{~F}$ tetrapolar catheter (Supreme 401475; SJM, Minnetonka, MN, USA) was situated in high right atria and through aortic valve a $4 \mathrm{~mm} 7 \mathrm{~F}$ ablation catheter (Livewire TC 402124; SJM, Minnetonka, MN, USA) was progressed to the left ventricle. In lateral mitral annulus, a continuous atrioventricular (AV) electrogram was registered (Fig. 2). One short radiofrequency $(\mathrm{RF})$ pulse programmed at $60 \mathrm{~W}$ and $60^{\circ} \mathrm{C}$

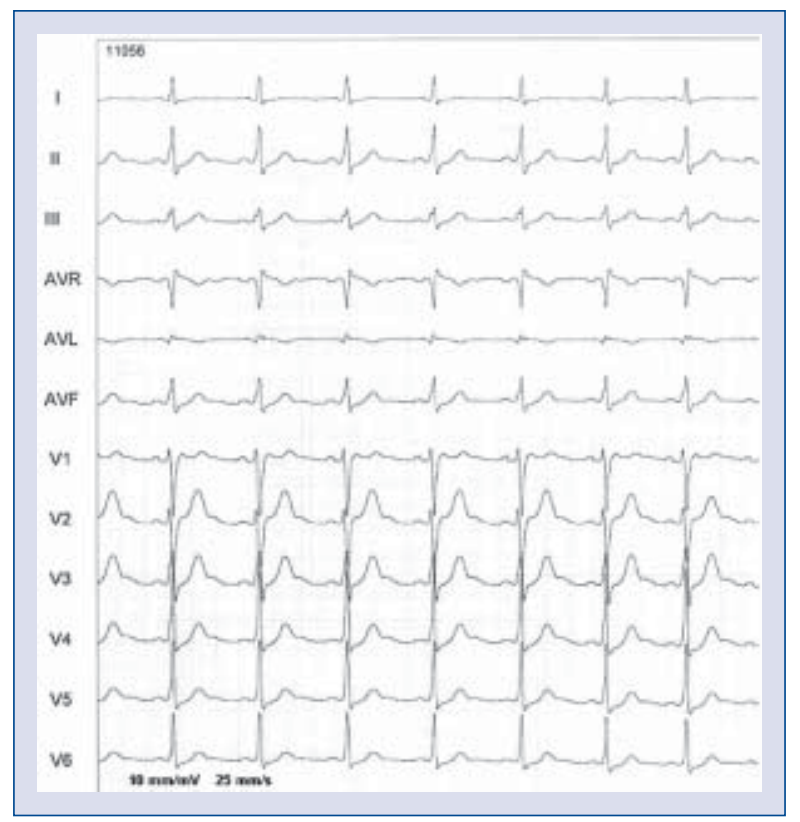

Figure 1. Basal electrocardiogram showing sinus rhythm and preexcitation with positive vector in $\mathrm{V} 1$ and III, suggesting left lateral pathway.

Address for correspondence: Antonio O. Luque Lezcano, MD, Intensive Care Medicine. Arrythmia Unit, Hospital Donostia, P. del Dr. Begiristain s/n, 20005 Donostia, Gipuzkoa (Basque Country), Spain, e-mail: oskar.luke@gmail.com 


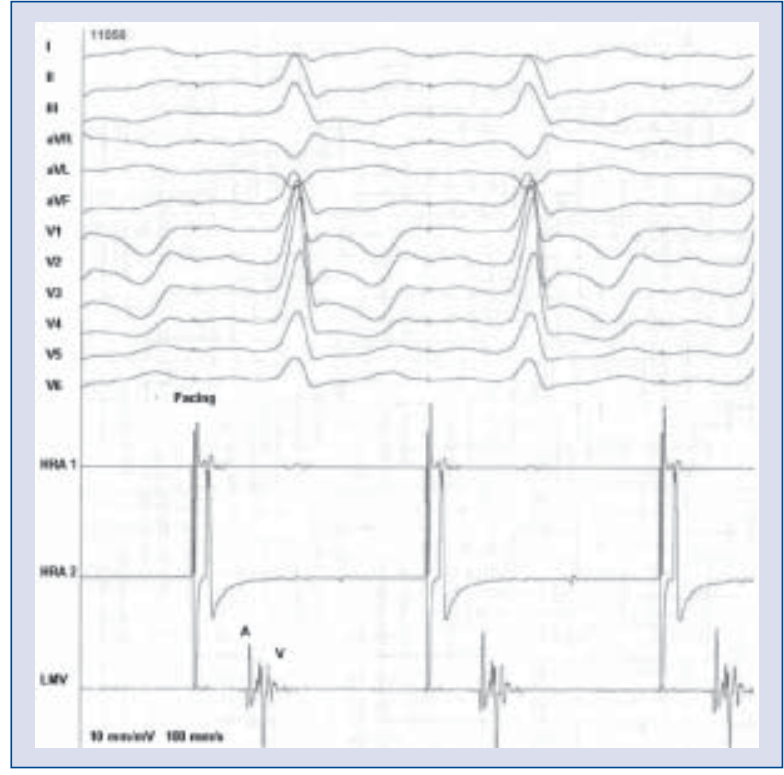

Figure 2. With atrial pacing, pre-excitation is highlighted and a continuous atrioventricular (AV) electrogram is registered in lateral mitral annulus.

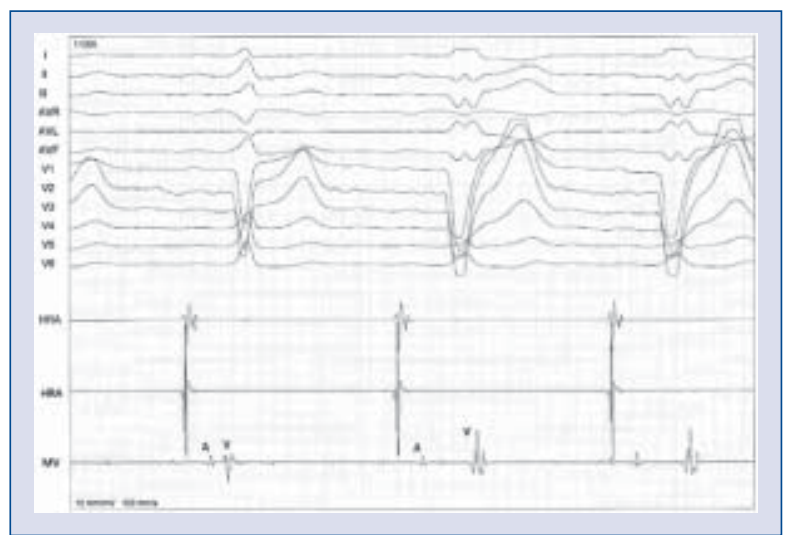

Figure 3. The first beat is last pre-excited QRS, and the next two are conducted through atrioventricular (AV) node with left bundle branch block. The $A$ and $V$ electrograms are separated when the pathway is blocked.

(Stockert ep shuttle; Biosense-Webster, Waterloo, Belgium) terminated pre-excitation and showed a complete left bundle branch block (LBBB). The pulse was stopped and preexcitation returned after some beats (Fig. 3). We considered the possibility of a transient LBBB, but the slow RR native cycle length of $774 \mathrm{~ms}$ and the fact that each of the seven times RF was delivered, the transient ablation of the accessory pathway showed the same LBBB, made it unlikely. With the movement of catheters,

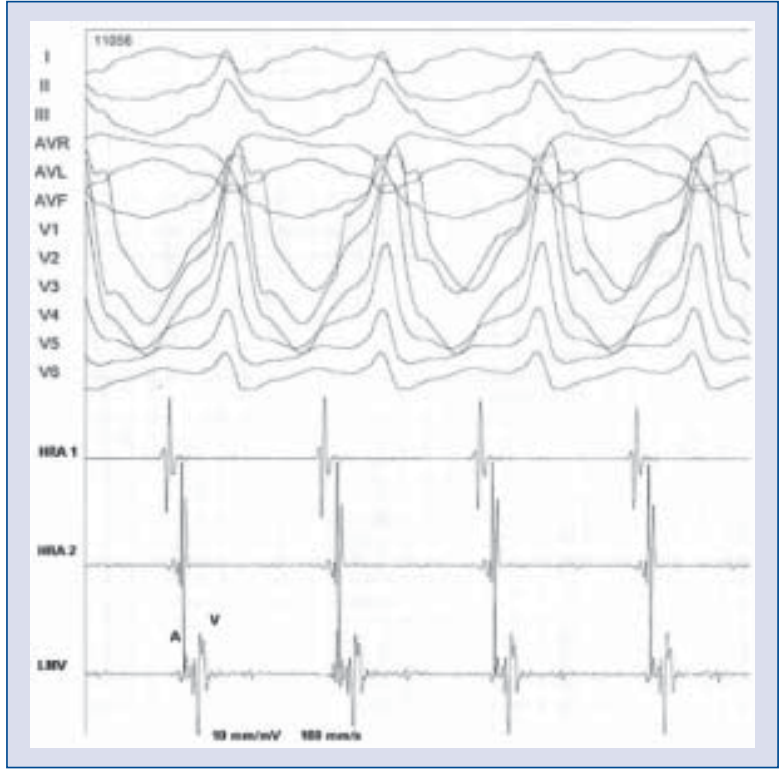

Figure 4. Antidromic tachycardia showing maximal preexcitation. Note $A$ and $V$ electrograms registered on the mitral annulus.

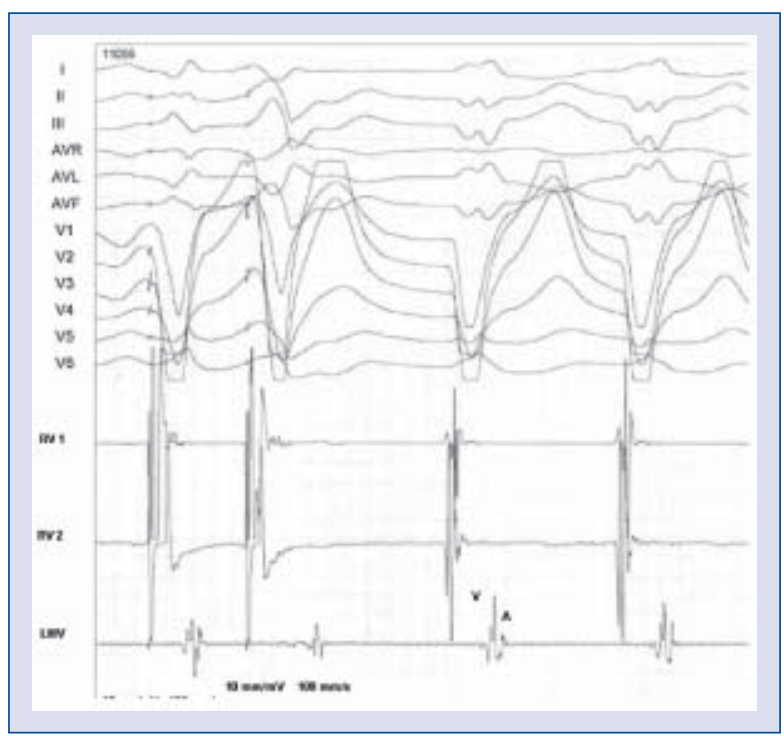

Figure 5. Initial two beats show the ventricular paced QRS that initiate an orthodromic tachycardia and complete left bundle branch block. Note the $\mathrm{V}$ and $\mathrm{A}$ electrograms registered on the lateral mitral annulus.

a $340 \mathrm{~ms}$ cycle length antidromic tachycardia was induced (Fig. 4). Two ventricular impulses initiated an orthodromic tachycardia with $420 \mathrm{~ms}$ cycle length (Fig. 5). Orthodromic tachycardia showed the same complete LBBB QRS as the one registered temporarily after the first RF pulse administered on 


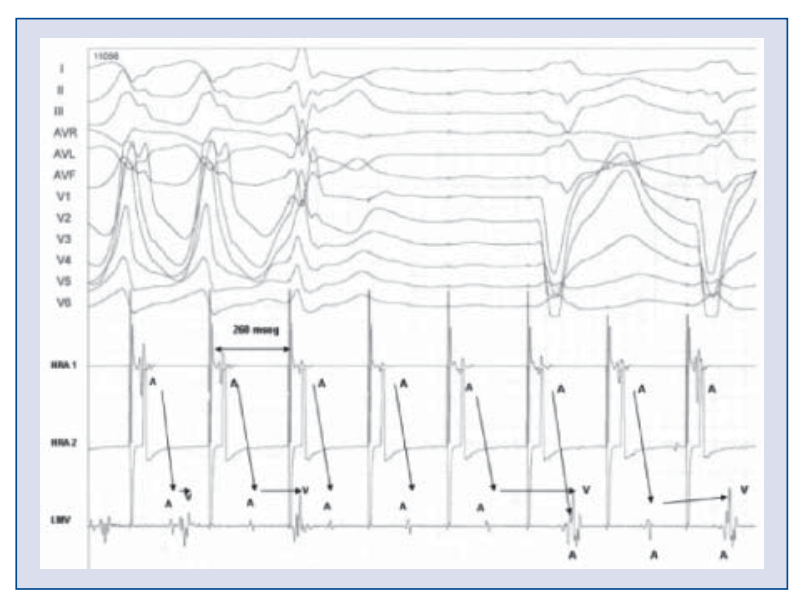

Figure 6. Effective refractory period of the pathway. Atrial pacing at 260 mseg blocks the pathway and a 2:1 nodal conduction with basal left bundle branch block starts. The fibers of the path probably get stunned and are unable to conduct at all.

the accessory pathway, reasonably demonstrating AV node conduction with complete LBBB (Fig. 6).

After assessing pros and cons, and in agreement with the patient, ablation was rejected.

\section{Discussion}

In Wolf-Parkinson-White syndrome, left lateral pathways are the most common. Their ablation is usually successful and tachycardias are definitely avoided by burning the abnormal branch of the re-entrant circuit.

The case we present has the singularity of a healthy young woman with an absence of conduction through the left branch of the heart, or maybe with complete absence of the left branch itself. Complete LBBB is an extremely rare finding among a young healthy population [1]. The 'normality' of the QRS is achieved by the left lateral pathway that operates as a 'prosthetic' left branch similar to the effect made by a resynchronization lead positioned on the left lateral ventricle in a patient with LBBB.

The long-term effect of LBBB in normal hearts is not well established. Clinical and echocardiographic follow-up of LBBB is mainly related to dilated cardiomyopathy and heart failure. However, there are some epidemiological studies that provide evidence of a worse outcome in a population affected with LBBB [2]. Perhaps in this case biventricular depolarization will prevent mechanical dyssyn- chrony and in the future an evolution to a dilated cardiomyopathy by ventricular remodeling [3-5].

Echocardiographic examination showed good synchronization measures between ventricles, intra left ventricle and also in AV relation: left ventricle pre-ejection interval (LVPEI) $136 \mathrm{~ms}$, LVPEI-right ventricle pre-ejection interval (RVPEI) $8 \mathrm{~ms}$, interventricular septum-posterior wall delay $32 \mathrm{~ms}$ diastolic filling time (mitral flow) related to heart cycle length $368 / 788 \mathrm{~ms}$ ( $>40 \%$ ) and normal tissue doppler imaging measures too: Q-septal and lateral time to onset of systolic velocity (132-136 ms) and septal and lateral post systolic shortening time (392-424 ms; 392-412 ms).

The sporadic crisis of palpitation due to re-entrant tachycardias are worth suffering in our judgement if we place on the other side of the scales the risk of a permanent heart dyssynchrony that could lead over the years to a dilated cardiomyopathy [5].

The fact that an atrial fibrillation might lead to syncope or, much less frequently, to sudden death, was also considered. Effective refractory period of the pathway in basal situation was $280 \mathrm{~ms}$, which was evaluated as secure enough to avoid sudden death in a healthy young woman.

We decided to maintain simultaneous biventricular depolarization notwithstanding the possibility of sporadic tachycardias. In our judgement, the alternative of a permanent LBBB for the rest of her life seemed more deleterious at that moment.

We are conscious that the decision can be discussed, and indeed that is the purpose of recording this case.

Conflict of interest: none declared

\section{References}

1. Siegman-Igra Y, Yahini JH., Goldbourt U, Neufeld HN. Intraventricular conduction disturbances: A review of prevalence, etiology, and progression for ten years within a stable population of Israeli adult males. Am Heart J, 1978; 96: 669-679.

2. Francia P, Balla C, Paneni F, Volpe M. Left bundle-branch block - pathophysiology, prognosis, and clinical management. Clin Cardiol, 2007; 30: 110-115.

3. Kass DA. Pathobiology of cardiac dyssynchrony and resynchronization. Heart Rhythm, 2009; 6: 1660-1665.

4. Stockburger M, Nitardy A, Fateh-Moghadam S et al. Electrical remodeling and cardiac dimensions in patients treated by cardiac resynchronization and heart failure controls. Pacing Clin Electrophysiol, 2008; 31: 70-77.

5. Barsheshet A, Moss AJ, McNitt S et al. Long-term implications of cumulative right ventricular pacing among patients with an implantable cardioverter-defibrillator. Heart Rhythm, 2011; 8: 212-218. 